\title{
Nuevas estrategias en trasplante de precursores hematopoyéticos
}

\author{
Mauricio Sarmiento Maldonado
}

Resumen: El trasplante hematopoyético es una estrategia terapéutica que permite posibilidad de curación en diversas enfermedades benignas y malignas. El autotrasplante tiene demostrada utilidad en mieloma y linfomas permitiendo recuperar la hematopoyesis luego de quimioterapias de alta intensidad. El alotrasplante permite reemplazar hematopoyesis defectuosa y/o introducir un potente efecto inmunológico llamado "efecto de injerto contra tumor". En los últimos años, se han desarrollado nuevos fármacos que permiten optimizar la recolección de progenitores autólogos y se han modificado los esquemas de trasplante, permitiendo un uso más amplio. El haplo trasplante alogénico ha favorecido que los enfermos tengan mejores posibilidades de encontrar donantes. En esta revisión, se analizan brevemente estas nuevas modalidades adoptadas en nuestro programa de trasplante hematopoyético.

Palabras clave: trasplante hematopoyético; trasplante autólogo; trasplante haploidéntico.

Abstract: Hematopoietic transplantation offers cure or control in several benign or malignant diseases. Autologous transplantation has proven to be useful in myeloma and lymphoma patients allowing hematopoiesis recovery after high-intensity chemotherapies. Allogeneic transplantation can replace defective hematopoiesis and / or introduce graft-versus-tumor effect. In recent years, new strategies have been developed to optimize autologous progenitor's collection and haploidentical modalities have allowed a wider use of allotransplants. In this brief review these new modalities adopted in our program are analyzed.

Keywords: Hematopoietic transplantation; autologous transplantation; haploidentical transplantation

Fecha de envío: 02 de agosto de 2016 - Fecha de aceptación: 30 de agosto de 2016

\section{Introducción}

Los primeros experimentos de Jacobson y colaboradores, en 1949 (Jacobson et al., 1949), mostraron que al infundir intraperitonealmente células de bazo en ratones irradiados, se lograba recuperar la hematopoyésis. Desde entonces el desarrollo del trasplante hematopoyético $(\mathrm{TH})$ ha avanzado continuamente (de La Morena et al., 2011). En la década del 1970, los experimentos fueron enfocados en probar el TH un mayor número de enfermedades hematológicas malignas. En años posteriores se aplicó con éxito el TH en enfermedades no malignas tales como errores innatos del metabolismo y hemoglobinopatías. Luego, en la década de 1990, la investigación mostró la posibilidad de trasplantar pacientes con quimioterapias preparatorias (acondicionamientos) de intensidad reducida, buscando tratar enfermos con estado funcional deteriorado o pacientes mayores. En los últimos 10 años, el avance en tratamientos antibióticos y antifúngicos de alta eficiencia y la optimización del soporte vital en unidades de cuidados intensivos, ha permitido que los pacientes sobrevivan al trasplante en mejores condiciones (Escobar et al., 2015). Esta dinámica de crecimiento en el conocimiento del TH ha sido mayor en los últimos años, con mejoría en los procesos de trasplante alogénico y autólogo. En este trabajo se describen algunos de los cambios que han tenido mayor impacto en nuestro programa de trasplante.

\section{Trasplante autólogo}

El trasplante autólogo hematopoyético (TAH) se fundamenta en que los pacientes afectos de enfermedades neoplásicas o autoinmunes puedan ser sometidos a tratamientos con quimioterapia de alta intensidad mieloablativa. Estos individuos sin la reinfusión de progenitores hematopoyéticos posterior no podrían sobrevivir por la insuficiencia medular permanente resultante. Así, los pacientes pueden recuperar su hematopoyesis por medio de reinfusión de progenitores autólogos en los casos con hemopatías malignas o eliminar los linfocitos autoreactivos, y reiniciar la formación linfocitaria en los casos con enfermedades autoinmunes (Garcia Lopéz et al., 2006). 
El trasplante autólogo de forma "clásica" se realiza con células progenitoras hematopoyéticas extraídas y crio-preservadas del paciente varios meses previos al tratamiento quimioterápico de control de la enfermedad de base que indica el trasplante. De esta manera, los pacientes afectos de mieloma (Brioli et al., 2016), linfomas (Reddy et al., 2014), leucemia aguda (Gorin, 2016) son derivados al programa de trasplante y luego de una exhaustiva evaluación de la función endocrina, cardíaca, respiratoria, metabólica, hepática, renal y de enfermedades infecciosas, son aceptados para acceder al TAH. Previa infusión subcutánea de filgrastim durante 5-6 días, por medio de aféresis se realiza la recolección de las células progenitoras (RPH) por vía periférica, necesarias para proceder con el trasplante con tiempos de recuperación de la hematopoyésis dentro de lo esperado. Una vez extraídas las células precursoras, son congeladas usando dimetil sulfóxido (DMSO). Este proceso es dispendioso en tiempo y recursos económicos y no está exento de riesgos para el paciente, pues se han descrito casos de toxicidad cardíaca y cerebral asociadas a la reinfusión de progenitores crio-preservados con DMSO (Zambelli et al.,1998, Mitrus et al., 2013).

La estimulación con filgrastim logra que el $60-80 \%$ de los pacientes tengan una RPH satisfactoria. Sin embargo, aproximadamente un $20 \%$ de los pacientes son primariamente refractarios a la RPH, y no logran recuentos celulares satisfactorios (Wu et al., 2016). Hay factores que predicen el fracaso de la RPH, incluyendo la exposición previa a quimioterapia de alta toxicidad o el uso previo de radioterapia. Estos pacientes "malos movilizadores" son un gran desafío, pues teniendo indicación perentoria de TAH, es difícil obtener sus células precursoras. La estrategia habitual es realizar varias $\mathrm{RPH}$, pudiendo no resultar recuentos satisfactorios.

En la actualidad, nuestro programa de trasplante ha incorporado una estrategia farmacológica para favorecer RPH exitosas, y ha modificado la forma clásica de TAH para evitar la crio-preservación. La primera estrategia ha sido incorporar el fármaco plerixafor en pacientes que tengan predictores de fracaso de recolección tales como: a. Mieloma múltiple tratado con melfalán, talidomida o lenalidomida; b. Mieloma o linfoma con más de 3 líneas de tratamientos; c. Falla de recolección previa; d. Menos de 30.000 CD34/ul de sangre periférica al día 5 de estimulación, e. Menos de 100.000 plaquetas el día de recolección y f. Antecedentes de radioterapia a zona pélvica. Este medicamento es un inhibidor del CXCR4, receptor expresado en las células progenitoras hematopoyéticas, relacionado con el homing medular. El uso del plerixafor favorece que las células progenitoras circulen en sangre periférica y puedan obtenerse $\mathrm{RPH}$ exitosas, sobre todo, en pacientes malos movilizadores. En nuestro programa se analizaron los pacientes que precisaron TAH entre el 2014 y el 2016, y se realizaron 55 recolecciones siendo
32 estimulados con filgrastim y 22 con filgrastim y plerixafor en pacientes malos movilizadores. El $100 \%$ de los procedimientos fueron exitosos en los dos grupos. No se encontraron diferencias significativas en cuanto a la RPH (estimulados con filgrastim: $1,56 \mathrm{RPH}$ y estimulados con filgastrim y plerixafor: $1.52 \mathrm{RPH}) \mathrm{ni}$ en la celularidad media obtenida (filgrastim: 3,9 CD34 x 10exp6/ $\mathrm{kg}$ y filgastrim y plerixafor 3.4 CD34 x 10exp6/kg). Este análisis mostró que con el uso racional del plerixafor se logran realizar $\mathrm{RPH}$ satisfactorias en pacientes con predictores de fracaso (datos aún no publicados).

La segunda estrategia fue modificar el esquema clásico de trasplante. Así, cuando los pacientes son enviados al programa de trasplante, son analizados por las mismas pruebas pretrasplante exhaustivas, pero la RPH no se hace en tiempo diferido, sino en fresco. Brevemente, una vez el paciente logra la mejor respuesta en el tratamiento de su enfermedad, es procesado con estimulación con filgrastim y/o plerixafor y se realiza la RPH; si esta resulta satisfactoria, ingresa inmediatamente al hospital e inicia su quimioterapia de acondicionamiento, recibiendo los precursores hematopoyéticos sin congelación ni manipulación con DMSO. Para esto hemos modificado la intensidad del tratamiento de acondicionamiento manteniendo las mismas dosis del esquema clásico, pero en forma condensada. Entre agosto de 2014 y julio de 2015, en nuestro programa se realizaron 18 trasplantes autólogos crio-preservados, y entre agosto de 2015 y abril de 2016 se han realizado 18 trasplantes con reinfusión sin crio-preservación. Los pacientes que recibieron trasplantes sin crio-preservación tuvieron media de prendimiento de injerto más precoz, menos neutropenia febril, nutrición parenteral y mucositis medida por la necesidad de uso de opiáceos, y menor estancia hospitalaria $(p<0.05)$ (Sarmiento et al. 2016).

\section{Trasplante alogénico}

El trasplante alogénico hematopoyético (AloTH) actualmente es la única estrategia curativa en enfermedades malignas hematológicas como las leucemias y linfomas agresivos. Su fundamento es reemplazar la hematopoyesis cancerosa e introducir un sistema inmune alogénico que provea efecto de injerto vs. tumor (Grosso et al., 2015). En otras enfermedades no malignas como las hemoglobinopatías, hemoglobinuria paroxística nocturna, aplasia medular o trastornos metabólicos heredados, se busca el cambio en la hematopoyesis enferma por una sana. Desde la década de 1970, con un mayor entendimiento del sistema HLA, hay más enfermedades que se benefician del trasplante, y ha aumentado el número de trasplantados a nivel mundial, principalmente de donantes hermanos. Sin embargo, dado el amplio polimorfismo 
de los genes que codifican el HLA, la posibilidad de encontrar un donante intrafamiliar es $25 \%$ y de donante no relacionado es $50 \%$ (Niederwieser et al., 2016). Así, antes de la década del 2000, casi la mitad de los pacientes no podían acceder a la realización de AloTH por la falta de donantes. La opción para aquellos pacientes sin donante intrafamiliar compatible fue realizar trasplantes alogénicos con sangre de cordón umbilical o donantes no relacionados HLA compatibles. El trasplante de cordón umbilical en adultos se asocia con prendimiento tardío, mayor incidencia de infecciones y elevados costos (Ramirez et al., 2011). El trasplante con donantes no relacionados es una buena estrategia, pero de elevado costo y con la dificultad agregada de que el tiempo necesario para encontrar un donante es de varios meses, lo cual puede permitir que la enfermedad de base recaiga y se pierda la oportunidad para el injerto. Nuestro programa fue pionero a nivel nacional en la realización de estas dos modalidades de alo trasplante pero dadas las dificultades descritas, fue necesario buscar otras estrategias. El trasplante haploidéntico se basa en la realización del procedimiento con donantes que no sean HLA idénticos, tales como padres, hermanos parcialmente compatibles, hijos o primos. Si bien años atrás se intentó la realización de trasplantes con donantes no compatibles o parcialmente compatibles, la mortalidad fue muy elevada, pues se usaron esquemas de profilaxis de enfermedad de injerto vs huéspedes clásicos (Beatty et al., 1985). El grupo de Seattle (Luznick et al., 2002, 2008) demostró que realizar trasplantes haploidénticos era posible al modificar la quimioterapia de acondicionamiento y profilaxis de EICH incorporando dosis altas de ciclofosfamida post trasplante. Si bien inicialmente se encontró en pequeños grupos de pacientes un mayor riesgo de recaída, datos recientes muestran que los resultados globales, incluyendo tasas de recaída, son por lo menos iguales a los obtenidos en trasplantes de donante hermano compatible y muy superiores a los trasplantes no relacionados y de cordón umbilical. Esto ha generado un incremento de potenciales donantes, permitiendo ofrecer trasplantes a más individuos.

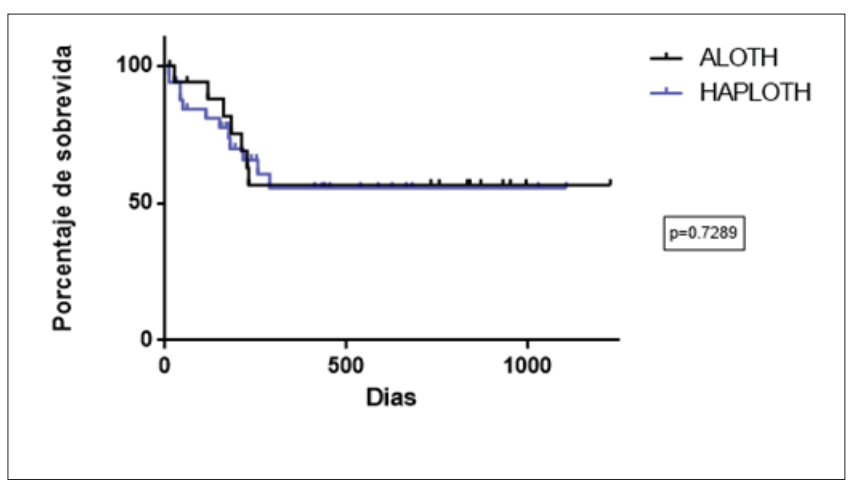

En el año 2012, nuestro programa de trasplante inició la implementación de la modalidad de AloTH haploidéntico logrando ajustar los procesos para iniciar esta modalidad. Desde enero de 2013 a la fecha se han realizado 33 trasplantes haploidénticos. Con una edad media de 35 años (rango 17-62 años) y con los siguientes diagnósticos: 13 pacientes con leucemia mieloide aguda, 9 con leucemia linfoblástica aguda, 1 con linfoma no Hodgkin de alto grado, 2 con aplasia medular, 2 con mielodisplasia grave, 3 con enfermedad de Hodgkin y 1 leucemia mieloide crónica. El $100 \%$ de los pacientes tuvieron prendimiento del injerto con recuperación plaquetaria al día 24 y granulocítica al día 16. La incidencia acumulada de $\mathrm{EICH}$ aguda a 100 días fue $17 \%$ y EICH crónica a 2 años fue 27\%. La sobrevida global a 2 años fue de $55 \%$ (figura 1). Estos resultados fueron comparados con pacientes de nuestro programa a quienes se les realizó trasplante de hermano $100 \%$ compatible, y no encontramos diferencias significativas en cuanto a mortalidad relacionada a trasplante y recaída (datos no publicados).

\section{Conclusiones}

El trasplante hematopoyético es en ocasiones la única opción curativa. Los avances en el proceso de trasplante y su implementación en nuestro programa han permitido que más personas puedan a optar a este tratamiento. El trasplante autólogo no crio-preservado junto con el uso de plerixafor, ha permitido trasplantes menos tóxicos. El trasplante haploidéntico ha favorecido que más pacientes tengan donantes intrafamiliares. Sin duda, en los próximos años se realizarán nuevos hallazgos que favorezcan una mejor tolerancia al trasplante con una mejor calidad de vida y sobrevida global de los enfermos afectos de graves condiciones benignas o malignas.

\section{Referencias bibliográficas}

Jacobson LO, Marks EK, Robson MJ, Gaston EO, Zirkle RE. Effect of spleen protection on mortality following X-irradiation. J Lab Clin Med 1949; 34: 1538-43.

De la Morena MT, Gatti RA. A history of bone marrow transplantation. Hematol Oncol Clin North Am. 2011 Feb;25(1):1-15.

Escobar K, Rojas P, Ernst D, Bertin P, Nervi B, Jara V, Garcia MJ, Ocqueteau M, Sarmiento M,Ramirez P. Admission of hematopoietic cell transplantation patients to the intensive care unit at the Pontificia Universidad Católica de Chile Hospital. Biol Blood Marrow Transplant. 2015 Jan;21(1):176-9.

García López A, Espigado Tocino I, Cid Boza N. Hematopoietic stem cell transplantation in systemic autoimmune diseases. State of the art. Reumatol Clin. 2006 May;2(3):146-51 
Brioli A. First line vs delayed transplantation in myeloma: Certainties and controversies.World J Transplant. 2016 Jun 24;6(2):321-30. Reddy NM, Perales MA. Stem cell transplantation in Hodgkin lymphoma. Hematol Oncol Clin North Am. 2014 Dec;28(6):1097-112.

Gorin C. Autologous stem cell transplantation versus alternative allogeneic donor transplants in adult acute leukemias.Semin Hematol. 2016 Apr;53(2):103-10.

Zambelli A, Poggi G, Da Prada G, Pedrazzoli P, Cuomo A, Miotti D et al. Clinical toxicity of cryopreserved circulating progenitor cells infusion. Anticancer Res. 1998 Nov-Dec;18(6B):4705-8.

Mitrus I, Smagur A, Giebel S, Gliwinska J, Prokop M, Glowala-Kosinska $M$ et al. A faster reconstitution of hematopoiesis after autologous transplantation of hematopoietic cells cryopreserved in $7.5 \%$ dimethyl sulfoxide if compared to $10 \%$ dimethyl sulfoxide containing medium. Cryobiology. 2013 Dec;67(3):327-31.

Wu CY, Chiou TJ, Liu CY, Lin FC, Lin JS, Hung MH et al. Decision-tree algorithm for optimized hematopoietic progenitor cell-based predictions in peripheral blood stem cell mobilization. Transfusion. 2016 May 27. [Epub ahead of print].

Sarmiento M, Parody R, Salas Q, Beffermann N, Jara V, Bertin P et al. Non-Cryopreserved autologous hematopoietic transplantation offers better quality of life during hospitalization period and similar transplant outcomes than cryopreserved mode in Myeloma and Lymphoma Patients. A Comparative Analysis of two centers. Drafting manuscript. Blood 2015.
Grosso DA, Hess RC, Weiss MA. Immunotherapy in acute myeloid leukemia. Cancer. 2015 Aug 15;121(16):2689-704.

Niederwieser D, Baldomero H, Szer J, Gratwohl M, Aljurf M, Atsuta $Y$ et al. Hematopoietic stem cell transplantation activity worldwide in 2012 and a SWOT analysis of the Worldwide Network for Blood and Marrow Transplantation Group including the global survey. Bone Marrow Transplant. 2016 Jun;51(6):778-85.

Ramirez P, Nervi B, Bertin P, Poggi H, Lagos M, Selman C, Pizarro I, jara $V$, Wiestruck A, Barriga F. Umbilical cord blood transplantation in hematologic diseases in patients over 15 years old: long-term experience at the Pontificia Universidad Católica de Chile. Transplant Proc. 2013: 45(10): 3734-9.

Beatty PG, Clift RA, Mickelson EM, Nisperos BB, Flournoy N, Martin PJ et al. Marrow transplantation from related donors other than HLA-identical siblings. N Engl J Med. 1985 Sep 26;313(13):765-71.

Luznik L, Engstrom LW, lannone R,Fuchs EJl. Post-transplantation cyclophosphamide facilitates engraftment of major histocompatibility complex-identical allogeneic marrow in mice conditioned with low-dose total body irradiation. Biol Blood Marrow Transplant 2002;8(3):131-8.

Luznik L, O'Donnell PV, Symons HJ, Chen AR, Leffell MS, Zahurak $M$ et al. HLA-Haploidentical bone marrow transplantation for hematologic malignancies using nonmyeloablative conditioning and high-dose, posttransplantation cyclophosphamide. Biol Blood Marrow Transplant 2008;14(6):641-50. 